\title{
Proinflammatory Extracellular Vesicle-Mediated Signaling Contributes to the Induction of Neuroinflammation in Animal Models of Endotoxemia and Peripheral Surgical Stress
}

\author{
F. Fricke ${ }^{1} \cdot$ J. Gebert ${ }^{1} \cdot$ J. Kopitz ${ }^{1}$ (D) K. Plaschke ${ }^{2}$
}

Received: 26 February 2020 / Accepted: 10 June 2020 / Published online: 18 June 2020

(c) The Author(s) 2020, corrected publication 2021

\begin{abstract}
Peripheral inflammation induced by endotoxemia or surgical stress induces neuroinflammation thereby causing neurological symptoms ranging from sickness behavior to delirium. Thus, proinflammatory signaling must be operative between the periphery and the central nervous system (CNS). In the present study, we tested whether nanometer-sized extracellular vesicles (EVs) that were produced during the peripheral inflammatory process have the capacity to induce neuroinflammation. Conditions of endotoxemia or surgical intervention were simulated in rats by lipopolysaccharide (LPS) injection or partial hepatectomy $(\mathrm{HpX})$. EVs were concentrated from these animals and tested for their proinflammatory action (I) in a microglial cell line and (II) by intracerebroventricular and (III) by intravenous injections into healthy rats. EVs from both conditions induced the secretion of cytokines from the glial cell line. Intracerebroventricular injection of the EVs caused the release of inflammatory cytokines to the cerebrospinal fluid indicating their pro-neuroinflammatory capacity. Finally, proinflammatory EVs were shown to pass the blood-brain barrier and induce neuroinflammation after their intravenous injection. Based on these data, we suggest that EV-associated proinflammatory signaling contributes to the induction of neuroinflammation in endotoxemia and peripheral surgical stress. Preliminary results suggest that peripheral cholinergic signals might be involved in the control of proinflammatory EV-mediated signaling from the periphery to the brain.
\end{abstract}

\section{Introduction}

Systemic inflammation, e.g., elicited by bacterial infection, large injuries or surgeries, induces physiological and neurological changes that are commonly referred to as 'sickness behavior' which is characterized by a decline of cognitive function, somnolescence, fever, decreased food intake and general fatigue. Since it is now quite clear that systemic inflammation can cause brain inflammation, such neuroinflammation is considered the main cause of the neurological changes ranging from sickness behavior to delirium

K. Plaschke

Konstanze.Plaschke@med.uni-heidelberg.de

1 Institute of Pathology, University Hospital Heidelberg, Im Neuenheimer Feld 224, 69120 Heidelberg, Germany

2 Department of Anesthesiology, University Hospital Heidelberg, Im Neuenheimer Feld 110, 69120 Heidelberg, Germany
(Poon et al. 2015). Consequently, the brain must be able to sense peripheral inflammation by communicating with the innate immune system. Several pathways of how the brain senses a peripheral inflammation have been suggested. For example, locally produced cytokines may activate primary afferent nerves such as the vagal nerves (Bluthe et al. 1994) thereby triggering signaling to the CNS (Chiu et al. 2013; Goehler et al. 2000). Also, circumventricular organs (CVO) are cerebral areas with incomplete endothelial blood-brain barrier (BBB) and therefore regarded as "gates to the brain". Thus, peripherally released proinflammatory cytokines may enter the CNS through this gate and elicit neuroinflammation (Wuerfel et al. 2010). Moreover, peripheral cytokines can gain access to the brain through specific transport systems in the BBB (Banks 2006). Finally, inflammatory damage to the microvascular endothelial cell monolayer that constitutes the luminal BBB surface, can lead to elevated BBB permeability which opens an access path for proinflammatory signaling molecules to the CNS (Rochfort and Cummins 2015).

Recently a novel mechanism for peripheral signaling to the CNS has been suggested. Based on the observation that extracellular vesicles are capable of passing the BBB, 
nominated them as potential vehicles to transfer peripheral signaling molecules to the brain (Chen et al. 2016; Matsumoto et al. 2017). The generic term "Extracellular Vesicles" (EVs) describes a heterogeneous population of lipid bilayerenclosed particles that are secreted by nearly all cells into the extracellular space (Becker et al. 2016; Mathieu et al. 2019; Witwer and Théry 2019). EVs can be classified into different EV subtypes according to their biogenesis and sizes. The two most prominent subclasses are exosomes ( 30 to $150 \mathrm{~nm}$ ) originating from the endosomal pathway and microvesicles $(100$ to $1000 \mathrm{~nm})$ that are plasma membrane-derived (Stahl and Raposo 2018). Circulating EVs are considered key players in cell-to-cell communication by transferring a molecular message encoded by their cargo to recipient cells (Raposo and Stahl 2019; van Niel et al. 2018; Wortzel et al. 2019). EVs are found in bodily fluids, such as blood, cerebrospinal fluid (CSF), saliva, and urine. Their cargo consists of nucleic acids, proteins, lipids and carbohydrates (van Niel et al. 2018). EVs present in the CSF have already been shown to be involved in signal transduction not only among neural cells but also among hematopoietic cells and in the peripheral nervous system (Kawahara and Hanayama 2018). Likewise, EVs in the brain play a role in CNS diseases, such as stroke, Alzheimer's disease, Parkinson's disease, prion disease, and traumatic encephalopathy, with both positive and negative effects (Liu et al. 2019). These pathologies of the brain are associated with a neuroinflammatory response. Neuroinflammation is an innate immune response induced by the microglia and astroglia, which respond to a proinflammatory signal by the production of cytokines, chemokines, reactive oxygen species and secondary messengers (Rama Rao and Kielian 2015). Various recent studies indicate that EVs may play a pivotal role in the initiation and control of neuroinflammation (Gupta and Pulliam 2014; Pascual et al. 2020). Therefore, various approaches have been applied to transfer anti-neuroinflammatory compounds through the BBB to attenuate the neuroinflammatory reactions that are considered to contribute to brain pathologies (Upadhya and Shetty 2019). On the other hand, the transport of proinflammatory EVs released during peripheral inflammation may also reach beyond the BBB and trigger neuroinflammation via glia activation ( $\mathrm{Li}$ et al. 2018). We addressed this question in two experimental animal models simulating conditions of endotoxemia and surgical stress. The experimental design is outlined in Fig. 1.

\section{Methods}

\section{Animals}

The study was performed on adult male Wistar rats (Janvier Labs from Saint-Berthevin Cedex, France), weighing
200-300 g. They were housed twice per cage in a temperature-controlled room at $22 \pm 0.5^{\circ} \mathrm{C}$ with a reversed daynight cycle (12 h:12 h, light on at 7 p.m.). Free access to food (LASQc diet, LA Svendi, Germany) and water was allowed throughout the experimental period. The experimental protocol was approved by the appropriate review committee of the Medical Faculty of the University of Heidelberg (Germany) and complied with the guidelines of the responsible national government agency (Regierungspraesidium Karlsruhe, Germany, G-104/18) and with international standards.

\section{Surgical Procedures}

Anesthesia was induced with 4 vol\% sevoflurane (Abbott, Wiesbaden, Germany) via a vaporizer (Dräger, Lübeck, Germany) and oxygen/nitrous oxide $\left(\mathrm{O}_{2}: \mathrm{N}_{2} \mathrm{O}=30: 70\right)$ and maintained over the entire experimental period with $3.5 \mathrm{vol} \%$ sevoflurane and oxygen/nitrous oxide $\left(\mathrm{O}_{2}: \mathrm{N}_{2} \mathrm{O}=30: 70\right)$. After endotracheal intubation with a $16-\mathrm{G}$ catheter, rats were ventilated with $60 / \mathrm{min}$ breathing frequency using a rodent respirator (Föhr Medical Instruments, Seeheim-Jugenheim, Germany). Tympanal temperature was monitored and maintained at around $37^{\circ} \mathrm{C}$ using a heating pad.

In all animals, catheters were placed in the arteria and vena femoralis sinistra (5.0 nylon thread) for administering drugs, for drawing blood samples, or for blood gas analysis. Partial hepatectomy ( $\mathrm{HpX}$ ) was conducted as described previously (Plaschke et al. 2018b). Lipopolysaccharide (LPS; $20 \mathrm{mg} / \mathrm{kg}$ body weight, from Escherichia coli O111:B4, LOT 028M4022V, Sigma- Aldrich, Munich, Germany) was slowly applied via the catheter in the vena femoralis sinistra (Plaschke et al. 2018a). Physostigmine as an inhibitor of the acetylcholine esterase was applied intravenously in a concentration of $0.04 \mathrm{mg} / \mathrm{kg}$ as detailed in a previous article (Plaschke et al. 2016).

For intraoperative blood analysis, blood samples $(0.1 \mathrm{ml}$ per sampling point) were taken via the arteria femoralis at 2 time points: before the surgical procedure was started (T0), and immediately before sacrifice (T1, final). Blood taken with a glass capillary $(10 \mu \mathrm{l})$ was immediately used to analyze blood [pH value, $\mathrm{pCO}_{2}, \mathrm{pO}_{2}$, bicarbonate $\left(\mathrm{HCO}_{3}{ }^{-}\right)$, base excess (BE), hematocrit (Hct), hemoglobin $(\mathrm{Hb})$, potassium $\left(\mathrm{K}^{+}\right)$, sodium $\left(\mathrm{Na}^{+}\right)$, glucose, and lactate concentrations].

After $4 \mathrm{~h}$ of LPS or $120 \mathrm{~min}$ after HpX, the animals were sacrificed under deep general anesthesia by bleeding out. The coagulated blood was centrifuged within $30 \mathrm{~min}$ at $7000 \mathrm{rpm}$ at $20{ }^{\circ} \mathrm{C}$ for $15 \mathrm{~min}$ and the supernatant was separated and frozen as serum at $-80{ }^{\circ} \mathrm{C}$ to assess biochemical parameters or for $\mathrm{EV}$ concentration. 


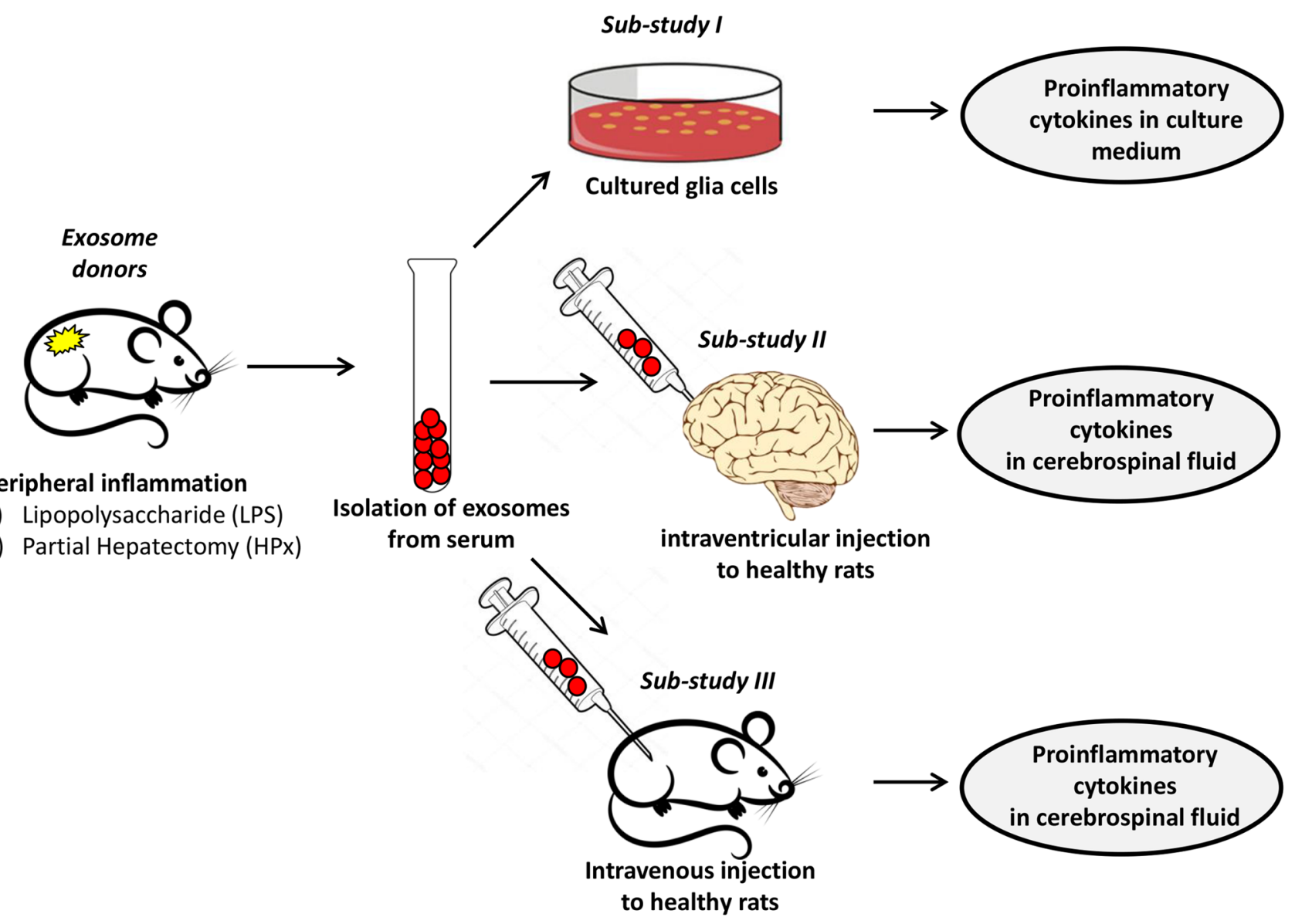

Fig. 1 Experimental design. Animals were treated either with LPS or by $\mathrm{HpX}$. Blood was sampled and EVs were isolated $4 \mathrm{~h}$ after LPS injection or $2 \mathrm{~h}$ after $\mathrm{HpX}$. EVs from untreated animals were taken as normal controls. Sub-study I: concentrated EVs were added to cultured glia cells and inflammatory cytokines assayed in the condi-

\section{Concentration and Characterization of EVs}

\section{Concentration}

Rat serum samples $(250 \mu \mathrm{l})$ were thawed at $37{ }^{\circ} \mathrm{C}$ and diluted 1:5 [v/v] with PBS (Thermo Fisher Scientific, Waltham, USA). Diluted samples were subjected to differential centrifugation (I. $5000 \times g, 15 \mathrm{~min}, 4^{\circ} \mathrm{C}$; II. $10,000 \times g$, $30 \mathrm{~min}, 4^{\circ} \mathrm{C}$ ). Cleared supernatants were transferred to sterile polycarbonate ultracentrifuge tubes and centrifuged at $120,000 \times g$ ( $k$-factor 34.8 ) for $120 \mathrm{~min}$ at $4{ }^{\circ} \mathrm{C}$ (TLA-100.2, rotor 100.2, Beckmann-Coulter, Krefeld, Germany). After discarding the supernatant, each EV pellet was resuspended in $20 \mu \mathrm{l}$ of PBS. EV preparations were stored at $-80^{\circ} \mathrm{C}$.

\section{Transmission Electron Microscopy}

EV samples were diluted 1:5 [v/v] with PBS and transferred onto 100 mesh formvar-coated copper grids. tioned medium was assayed after 1 h. Sub-study II: EVs were administered by an intracerebroventricular (icv) injection to healthy rats. CSF was taken $1 \mathrm{~h}$ after the injection and assayed for inflammatory cytokines. Sub-study III: EVs were administered by an intravenous (i.v.) injection via the femoral vein to healthy rats

Negative staining was performed with $2 \%$ aqueous uranyl acetate. Air-dried samples were visualized at $80 \mathrm{kV}$ using a JEM 1400 transmission electron microscope (JOEL USA, Peabody, USA) equipped with a Tietz 2 K digital camera.

\section{Nanoparticle Tracking}

Nanoparticle tracking analysis was performed with the ZetaView PMX110 system and the software 8.04.02 SP2 (Particle Metrix, Inning, Germany) according to the manufacturer's instructions. Measurements were performed at 11 different positions in a dilution of $1: 10,000[\mathrm{v} / \mathrm{v}]$ with PBS. Settings for data acquisition were adjusted to a sensitivity of $95 \%$, a shutter of 100 , and a frame rate of 30 frames per second. 


\section{Protein Extraction and Western Blot}

Proteins were extracted in RIPA buffer $(50 \mathrm{mM}$ Tris- $\mathrm{HCl}$ pH 7.4, $150 \mathrm{mM} \mathrm{NaCl}, 1 \%$ Triton X-100, 1\% Na-deoxycholate, $0.1 \% \mathrm{SDS}, 0.1 \mathrm{mM} \mathrm{CaCl}_{2}$ and $0.01 \mathrm{mM} \mathrm{MgCl}_{2}$ ) supplemented with cOmplete Mini protease inhibitor cocktail inhibitor (Roche, Basel, Switzerland). Protein concentrations were measured by Bradford assay (Bio-Rad Laboratories, Hercules, USA) following the manufacturer's instructions. Per sample, $20 \mu \mathrm{g}$ (CD63, CD9, Alix) or $40 \mu \mathrm{g}$ (ApoA1) of protein were separated on 4-20\% Bis-Tris gradient gels (Expedeon, Cambridge, UK) and blotted onto a nitrocellulose membrane (Life Technologies, Carlsbad, Germany). After blocking the membrane, the following primary antibodies were used: anti-CD63 (1:300, MX-49.129.5, Santa Cruz, Heidelberg, Germany), anti-CD9 (1:200, C4, Santa Cruz), and goat anti-Alix (1:800, Q19, Santa Cruz), rabbit anti-ApoA1 (1:530, polyclonal ab20453, Abcam). Primary antibodies were diluted in 5\% milk/TBST (CD63, CD9, Alix) or in PBS/Tween-20 (ApoA1) and incubated with membranes overnight at $4{ }^{\circ} \mathrm{C}$. The blots were washed with TBST and incubated with a sheep anti-mouse-IgG HRP (1:5000; GE-Healthcare, Chicago, USA), donkey anti-goatIgG HRP (1:1000; Santa Cruz) or a goat anti-rabbit-IgG HRP (1:2000; Promega, Madison, USA) secondary antibody for $1 \mathrm{~h}$ at room temperature. Signals were detected using Western Lightning Plus ECL (Perkin Elmer, Waltham, USA) and a ChemiDoc MP System (Bio-Rad Laboratories, Hercules, USA).

\section{Availability of Data and Materials}

We have submitted all relevant data of our experiments to the EV-TRACK knowledgebase (EV-TRACK ID: EV200028) (Van Deun et al. 2017).

\section{Routes of EV Application}

\section{Cell Culture}

Human microglial HMC3 cells (Dello Russo et al. 2018) were obtained from LGC Standards GmbH (Wesel, Germany) and cultured in 96 well plates with DMEM medium containing 10\% fetal calf serum (Sigma-Aldrich, Taufkirchen, Germany) and $1 \%$ penicillin/streptomycin. At $80 \%$ confluency, two concentrations (10 and $50 \mu \mathrm{g} / \mathrm{ml}$ ) of $\mathrm{EV}$ preparations were added to the medium for $1 \mathrm{~h}$ before the medium was used for biochemical assays.

\section{Intracerebroventricular (icv) Injection}

Rats underwent inhalational anesthesia with 3.5 vol\% sevoflurane and 30:70 $\mathrm{O}_{2}: \mathrm{N}_{2} \mathrm{O}$ and via a rat-adapted mask. $10 \mu \mathrm{l}$ of a suspension of $\mathrm{EVs}$ in sterile $0.9 \% \mathrm{NaCl}$ (corresponding to $10 \mu \mathrm{g}$ protein) was injected icv into the brain of healthy rats $(n=6)$. The icv injection procedure was performed as described in detail in a previous study (Plaschke and Hoyer 1993). One hour after icv injection, the cerebral spinal fluid (CSF) was taken (50-200 $\mu \mathrm{l}$ per rat) between the cervical vertebras via a small syringe. In addition, blood was drawn from the femoral vein as described above. Both fluids were immediately stored at $-80{ }^{\circ} \mathrm{C}$ before further biochemical analysis.

\section{Intravenous (i.v.) Injection}

Rats underwent inhalational anesthesia with 3.5 vol\% sevoflurane and 30:70 $\mathrm{O}_{2}: \mathrm{N}_{2} \mathrm{O}$ via a rat-adapted mask. The femoral vein was catheterized. $500 \mu \mathrm{l}$ of a suspension of EVs (corresponding to $250 \mu \mathrm{g}$ protein) was injected i.v. into $n=6$ healthy rats. One hour after i.v. injection, CSF was taken (50-200 $\mu$ l per rat) between the cervical vertebras via a small syringe. In addition, blood was taken via the femoral vein as described above. Both fluids were immediately stored at $-80^{\circ} \mathrm{C}$ before further biochemical analysis.

\section{Fluorescent Labeling of EVs}

Carboxyfluorescein succinimidyl ester (CFSE) was used for sensitive fluorescent labeling of EVs. In particular, EVs $(200 \mu \mathrm{g})$ were suspended in $100 \mu \mathrm{l} \mathrm{PBS} / 0.5 \%$ BSA. Subsequently, $100 \mu \mathrm{l}$ of $20 \mu \mathrm{M}$ CFSE in PBS/0.5\% BSA was added and the mixture incubated for $30 \mathrm{~min}$ at $37{ }^{\circ} \mathrm{C}$ in the dark. The reaction was stopped by the addition of $400 \mu \mathrm{l}$ RPMI medium. CFSE-labeled EVs were washed and collected by ultracentrifugation as described above. For their detection in CSF, fluorescence was measured at Ex $=492 \mathrm{~nm}$ and $\mathrm{Em}=517 \mathrm{~nm}$.

\section{Biochemical Assays}

The concentration of proinflammatory cytokines was determined in plasma samples and CSF using the following ELISA kits: Quantikine Rat TNF- $\alpha$ (RTA00), interleukin-6 (IL6; R6000B), and interleukin-1 $\beta$ (IL-1B; RLB00) (R\&D Systems, Wiesbaden, Germany). All analyses were performed according to the manufacturer's instructions.

\section{Statistics}

All analyses were performed by an independent investigator blinded to the experimental conditions. Data in figures were presented as mean values \pm standard deviation (S.D.). Differences between the groups (HpX versus control and LPS versus control) within normally distributed data were subjected to one-way ANOVA with following post hoc Tukey 
test using SPSS v22.0 (SPSS IBM, Chicago, USA). Results were considered significant at $p \leq 0.05$.

\section{Results}

In order to simulate severe infection, rats were treated with an intravenous injection of lipopolysaccharide (LPS). Partial hepatectomy $(\mathrm{HpX})$ was used to reflect a typical surgical intervention without an organ-specific deterioration (that means without significant changes in albumin and liver enzymes). The HpX model has been described in detail previously (Plaschke et al. 2018b).
Before administering LPS or performing $\mathrm{HpX}$, no significant differences between the groups were observed for the following blood parameters: $\mathrm{pH}$ value, $\mathrm{pCO}_{2}, \mathrm{pO}_{2}$, bicarbonate $\left(\mathrm{HCO}_{3}{ }^{-}\right)$, base excess (BE), hematocrit ( $\left.\mathrm{Hct}\right)$, hemoglobin $(\mathrm{Hb})$, potassium $\left(\mathrm{K}^{+}\right)$, sodium $\left(\mathrm{Na}^{+}\right)$, glucose (Glu), and lactate (Lac) concentrations (data not shown). In untreated animals, all these measured parameters were in the physiological range. However, after treatment significant changes were found in $\mathrm{pH}$ value, $\mathrm{K}^{+}, \mathrm{Na}^{+}$, base excess, bicarbonate, glucose, and lactate concentration in endotoxemic (LPS-treated) rats compared to saline-injected control rats $\left({ }^{*} p \leq 0.05\right.$, Fig. $\left.2 \mathrm{a}\right)$. Partial hepatectomy (HpX), however, did not induce significant changes in blood parameters, except for blood glucose concentration (Fig. 2a).

a Blood analysis

\begin{tabular}{|c|c|c|c|c|c|c|c|c|c|c|c|}
\hline & $\mathrm{pH}$ & $\begin{array}{l}\mathrm{pCO}_{2} \\
(\mathrm{~mm} \mathrm{Hg})\end{array}$ & $\begin{array}{c}\mathrm{pO}_{2} \\
(\mathrm{~mm} \mathrm{Hg})\end{array}$ & $\begin{array}{l}\mathrm{HCO}_{3}^{-} \\
(\mathrm{mmol} / \mathrm{l})\end{array}$ & $\begin{array}{c}\mathrm{BE} \\
(\mathrm{mmol} / \mathrm{l})\end{array}$ & $\begin{array}{l}\text { Hct } \\
(\%)\end{array}$ & $\begin{array}{l}\mathrm{Hb} \\
(\mathrm{g} / \mathrm{l})\end{array}$ & $\begin{array}{c}\mathrm{Na}^{+} \\
(\mathrm{mmol} / \mathrm{l})\end{array}$ & $\begin{array}{c}\mathrm{K}^{+} \\
(\mathrm{mmol} / \mathrm{l}) \\
\end{array}$ & $\begin{array}{c}\text { Glucose } \\
(\mathrm{mg} / \mathrm{dl})\end{array}$ & $\begin{array}{c}\text { Lactate } \\
(\mathrm{mmol} / \mathrm{l})\end{array}$ \\
\hline \multirow[t]{2}{*}{ Control } & 7,47 & 34,5 & 121,1 & 24,7 & 1,52 & 39,0 & 13,25 & 139,1 & 4,26 & 194,6 & 1,62 \\
\hline & $\pm 0,03$ & $\pm 4,23$ & $\pm 17,24$ & $\pm 3,16$ & $\pm 2,92$ & $\pm 6,29$ & $\pm 2,23$ & $\pm 2,14$ & $\pm 0,30$ & $\pm 23,25$ & $\pm 0,84$ \\
\hline \multirow[t]{2}{*}{ LPS } & 7,02 & 59,8 & 79,8 & 14,3 & $-16,5$ & 32,8 & 11,2 & 144,9 & 8,52 & 19,8 & 6,46 \\
\hline & $\pm 0,14^{*}$ & $\pm 15,6$ & $\pm 21,1$ & $\pm 2,5^{*}$ & $\pm 4,58^{*}$ & $\pm 5,2^{*}$ & $\pm 1,9^{*}$ & $\pm 4,7^{*}$ & $\pm 1,5^{*}$ & $\pm 44,27^{*}$ & $\pm 2,35^{*}$ \\
\hline \multirow{3}{*}{$\begin{array}{l}p \\
\text { (LPS vs. control) } \\
\mathrm{HpX}\end{array}$} & 0,007 & 0,08 & 0,106 & 0,005 & 0,001 & 0,01 & 0,044 & 0,010 & 0,0008 & 0,0001 & 0,0081 \\
\hline & 7,44 & 41,5 & 141,5 & 26,5 & 2,6 & 37,2 & 12,5 & 137,6 & 4,46 & 145,1 & 1,46 \\
\hline & $\pm 0,03$ & $\pm 4,0$ & $\pm 18,9$ & $\pm 1,4$ & $\pm 1,2$ & $\pm 2,9$ & $\pm 1,0$ & $\pm 1,6$ & $\pm 0,6$ & $\pm 19,8^{*}$ & $\pm 0,3$ \\
\hline $\begin{array}{l}p \\
\text { (HpX vs. control) }\end{array}$ & n.s. & n.s. & $n . s$ & n.s. & n.s. & n.s. & n.s. & n.s. & n.s. & 0,01 & n.s. \\
\hline
\end{tabular}

*: $p \leq 0,05 ;$ n.s. not significant

\section{b Inflammatory cytokines}
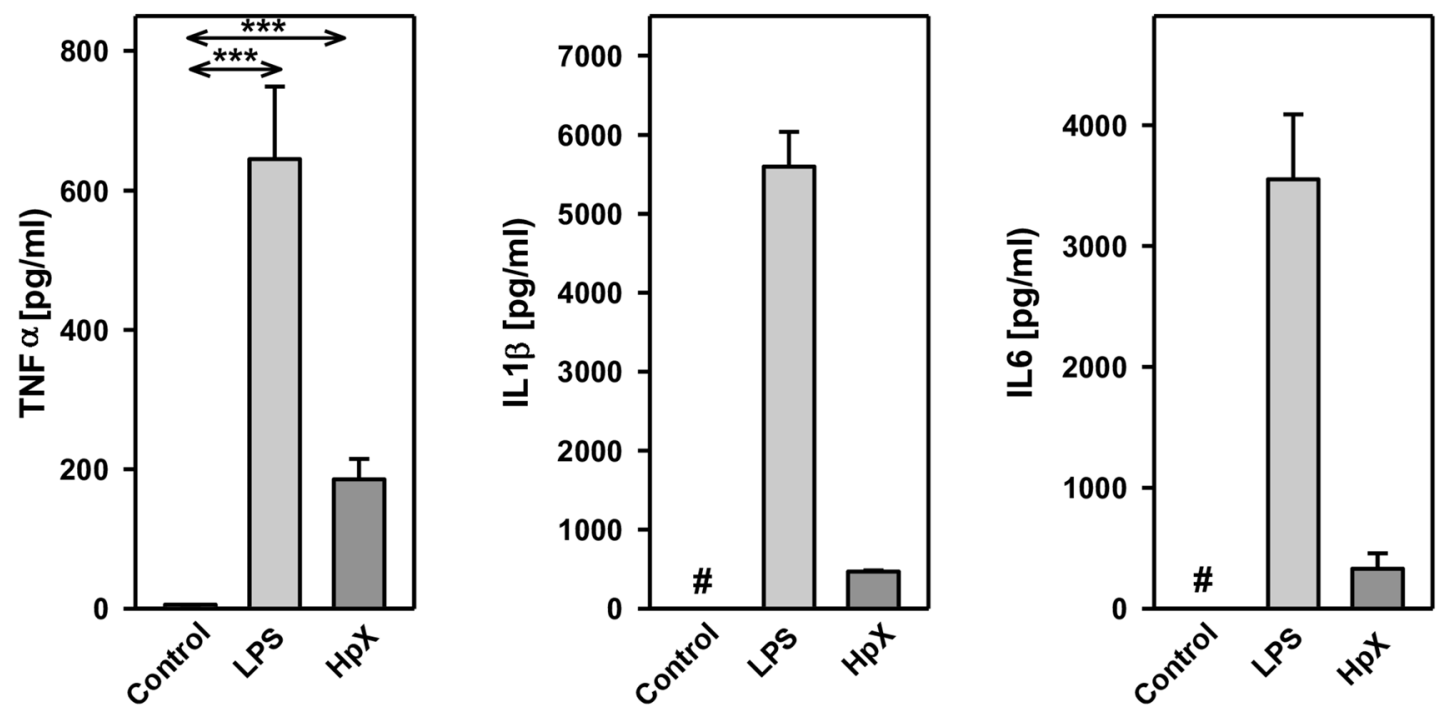

Fig. 2 Blood parameters and cytokine release in LPS- and HpXtreated rats. A total of 18 adult male rats were randomly allocated to 3 groups with $n=6$ per group. Control group was injected with $0.9 \% \mathrm{NaCl}$, LPS group received an injection of LPS $(20 \mathrm{mg} / \mathrm{kg}$ body weight in $0.9 \% \mathrm{NaCl}$ ), $\mathrm{HpX}$ group was subjected to partial $\mathrm{HpX}$. Blood was sampled $4 \mathrm{~h}$ after LPS and placebo injection, and $2 \mathrm{~h}$ after $\mathrm{HpX}$. a Blood parameters were recorded at the end of the experimental period. $L P S$ lipopolysaccharide, $H p X$ partial hepatectomy, $B E$ base excess, $H c t$ hematocrit, $H b$ hemoglobin, n.s. not significant. *Significant effect ( $p<0.05$, one-way ANOVA with following post hoc Tukey test) between LPS or HpX versus control. b Inflammatory cytokines in the rats' serum (obtained from the blood) at the end of the experimental period. Results are means of each group. \#Below detection limit of the assay. Error bars indicate S.D. ${ }^{* *} p<0.001$. In the case that values were below detection limit of the assay, no statistical analysis could be performed 
The inflammatory cytokines TNF- $\alpha$, interleukin- $1 \beta$ (IL1ß) and interleukin-6 (IL6) were strikingly increased in LPS- and HpX-treated animals as compared to untreated controls indicating peripheral inflammation caused by endotoxemia or surgical treatment. The inflammatory reaction was more pronounced in the endotoxemic rats (Fig. 2b). EVs were then concentrated from both treatment groups (LPS, $\mathrm{HpX}$ ) and untreated controls by differential ultracentrifugation and characterized by three different approaches (Thery et al. 2018). First, EV preparations were analyzed by negative stain transmission electron microscopy (TEM) to visualize the morphology and size of individual vesicles. As exemplified in Fig. 3a, lipid bilayer-enclosed particles that ranged in diameter from 30 to $150 \mathrm{~nm}$ were detected as round- and cup-shaped structures in all preparations. Second, nanoparticle tracking analysis (NTA) confirmed the concentration and size distribution of isolated particles. The majority of the yield ranged in a monopeak-fashion from 30 to $150 \mathrm{~nm}$, resulting in mean diameters of $106.2 \pm 43.4 \mathrm{~nm}$ (median $88.8 \mathrm{~nm}$ ) for LPS samples or $109.0 \pm 49.3 \mathrm{~nm}$ (median $91.7 \mathrm{~nm}$ ) for HpX samples (Fig. 3b). Third, western blot (WB) analysis revealed the expression of three EV marker proteins in EV protein lysates (Fig. 3c). The two transmembrane proteins CD63 and CD9 as well as the cytosolic protein Alix were found to be expressed in EV protein lysates. Further, WB analysis also revealed the presence of apolipoprotein A1 (ApoA1) in all preparations (Fig. 3c), indicating that other extracellular particles, such as lipoproteins, were either co-sedimented or associated with EVs from rat serum samples. Taken together, our results demonstrate the successful enrichment of extracellular vesicles and particles from rat serum samples and no substantial difference was detected between all EV bulk preparations. The defined EV preparations were used for all further investigations. In order to test their direct effect on glia cells, HMC3 cells were treated with two concentrations of EVs (Fig. 1/sub-study I). EVs concentrated from LPS-treated animals strikingly induced the release of inflammatory cytokines from these cells (Fig. 4a). Likewise, EVs from HpX-treated animals also elicited the release of TNF- $\alpha$ and IL6, but at lower potency compared to EVs from LPS animals. No effect of HpX EVs on IL1ß secretion was detectable (Fig. 4b). Altogether, these results reflect the stronger inflammation in the LPS animals from which the EVs were isolated. In the next step, the in vivo action of the isolated EVs (LPS, HpX) was tested (Fig. 1/sub-study II). To this end, these EV preparations were injected intracerebroventricularly into the brain of healthy rats. LPS- and HpX-derived EVs induced the release of TNF- $\alpha$, IL1ß and IL6 to the CSF (Fig. 5), indicating that proinflammatory EVs from the periphery have the potential to induce inflammation in the brain. This raised the question whether EVs in the periphery can pass the blood-brain barrier (BBB) thereby inducing neuroinflammation. To address this issue, we injected LPS- and HpX-induced EVs into a a
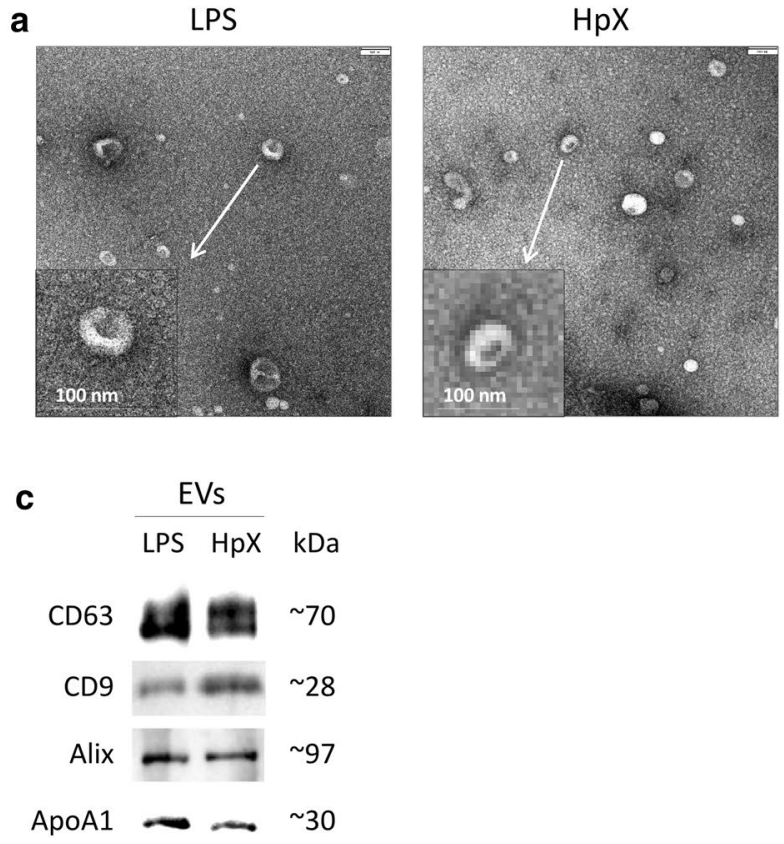

Fig. 3 Characterization of rat serum-derived EVs. a Negative stain transmission electron microscopy revealed the size and shape of EVs concentrated from LPS- and HpX-treated rats. Scale bar $100 \mathrm{~nm}$. b Nanoparticle tracking analysis determined the size distribution b

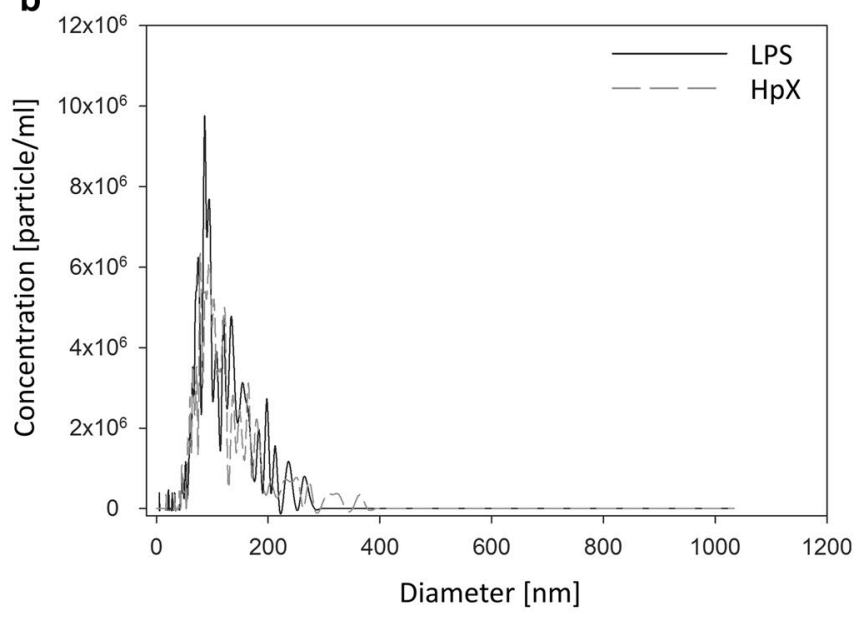

(x-axis) and particle concentration (y-axis) of the isolated yield. c Western blot analysis showed EV protein marker (CD63, CD9, Alix) and high-density lipoprotein marker (ApoA1) expression. Molecular weights $(\mathrm{kDa})$ of each protein are indicated 
Fig. 4 Effect of LPS- or HpXderived EVs on cultured glia cells. EVs from LPS- (a) or $\mathrm{HpX}$ - (b) treated animals were added to the culture medium of HMC3 glia cells at the indicated concentrations. After $1 \mathrm{~h}$ the conditioned medium was assayed for TNF- $\alpha$, IL1ß and IL6. Results are the means of 6 independent observations. Error bars indicate S.D. \#Below detection limit of the assay. $* * * p<0.001$. In the case that values were below detection limit of the assay, no statistical analysis could be performed
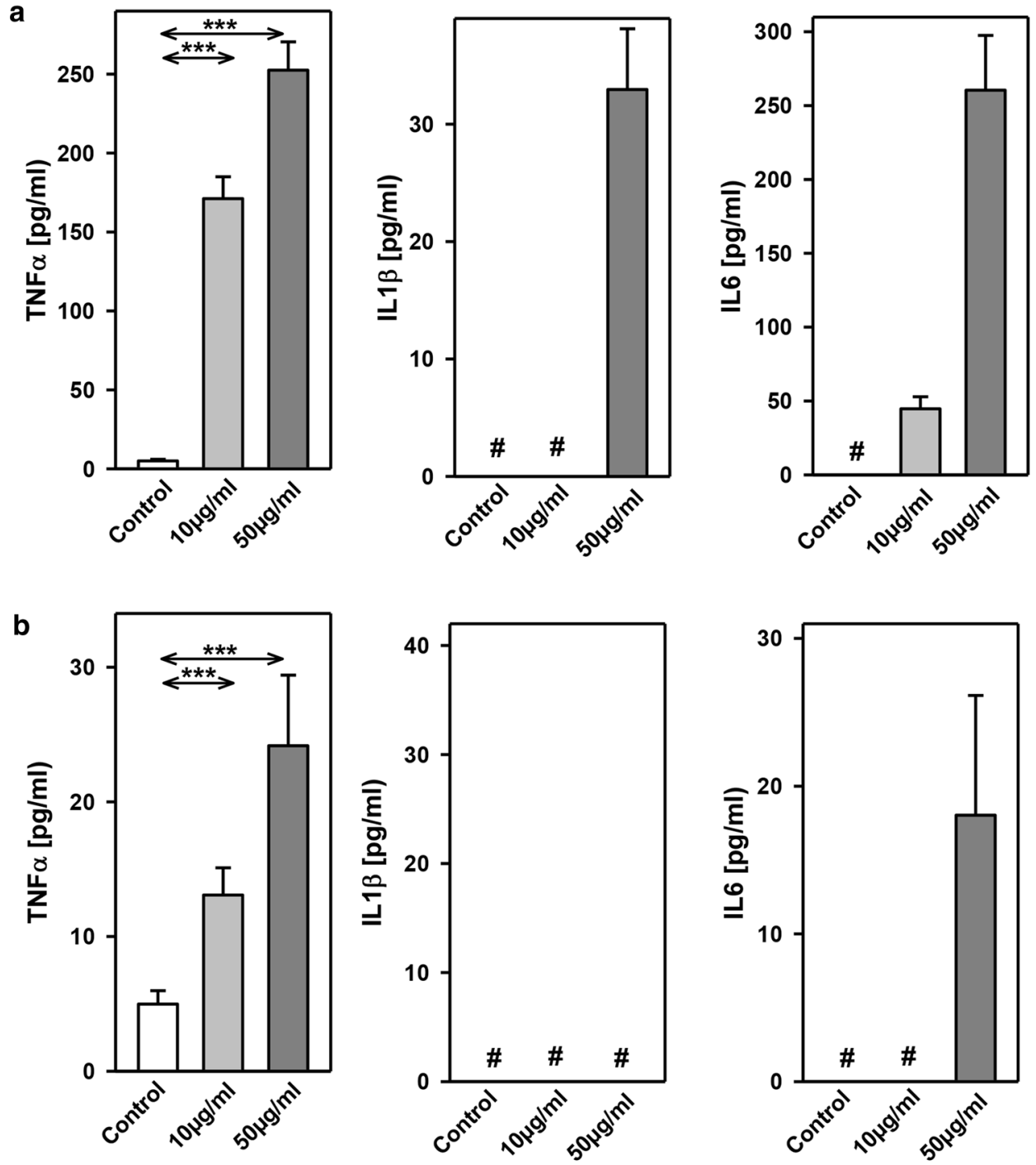

Fig. 5 Cytokine release to the $\mathrm{CSF}$ after intracerebroventricular injection of LPS- or HPxderived EVs. EVs $(10 \mu \mathrm{g})$ from LPS- or HpX-treated animals were administered by intracerebroventricular (icv) injection to healthy rats. After $1 \mathrm{~h}$ CSF was taken and assayed for TNF- $\alpha$, IL1ß and IL6. Results are the means of 6 independent observations. Error bars indicate S.D. $* * * p<0.001$

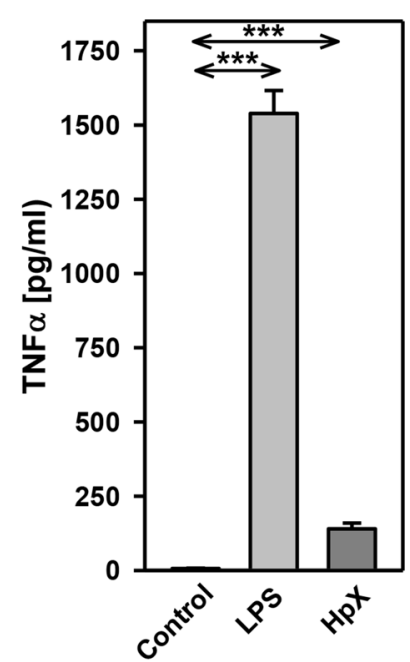

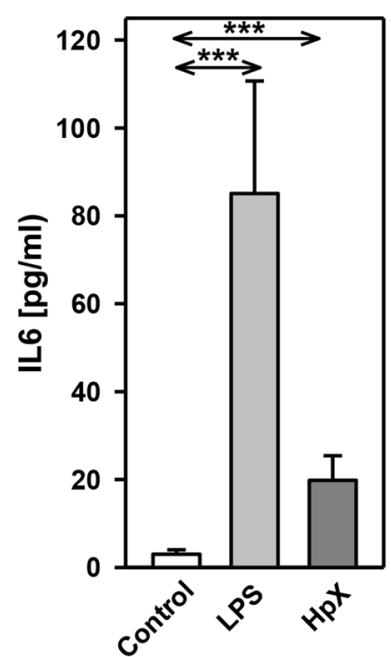


Fig. 6 Cytokine release to the CSF after peripheral intravenous injection of LPS- or HpXderived EVs. EVs $(250 \mu \mathrm{g})$ from LPS- or HpX-treated animals were administered by intravenous injection via the femoral vene to healthy rats. After $1 \mathrm{~h}$ CSF was taken and assayed for TNF- $\alpha$, IL1ß and IL6. Results are the means of 6 independent observations. Error bars indicate S.D. \#Below detection limit of the assay. $* * * p<0.001$. In the case that values were below detection limit of the assay, no statistical analysis could be performed
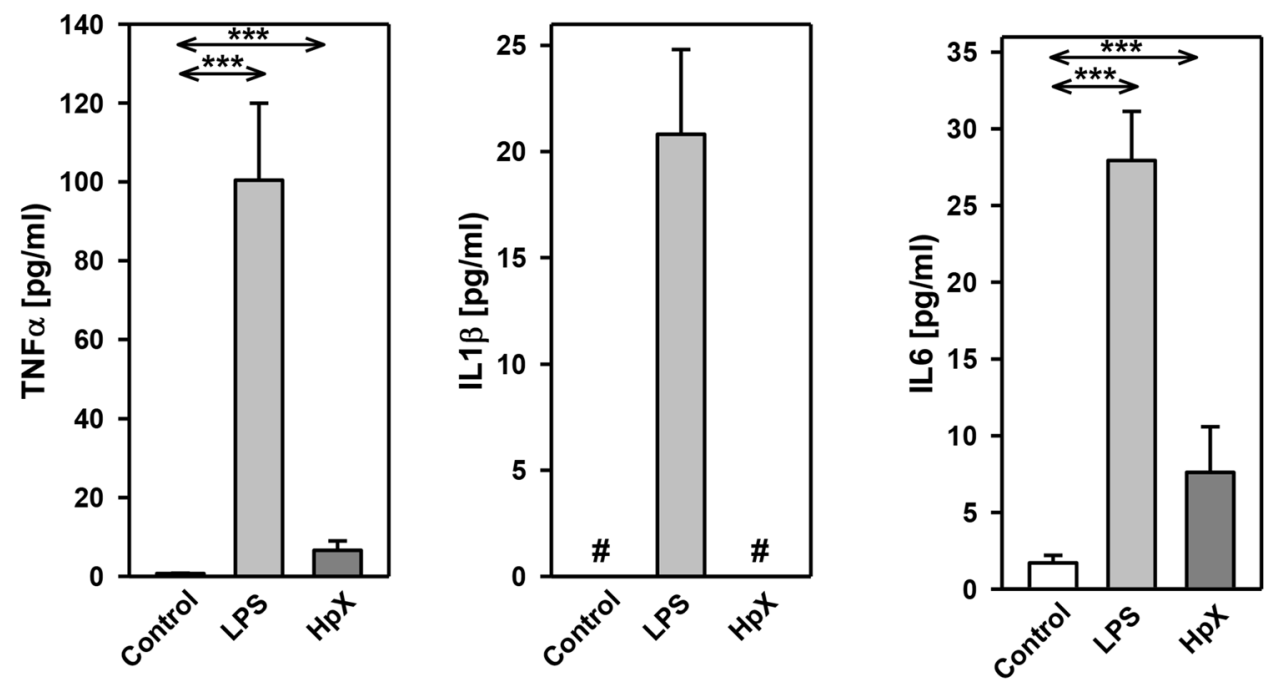

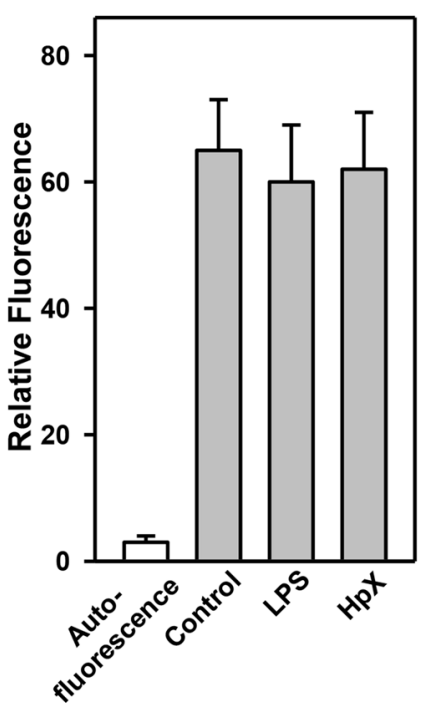

Fig. 7 Detection of peripherally injected EVs in the CSF. EVs from healthy animals were fluorescein-labeled with CSFE and administered by intravenous injection via the femoral vein to LPS-, HpXtreated or control animals as described in Fig. 2. After $1 \mathrm{~h}$ CSF was taken and fluorescence measured. Results are the means of 3 measurements. Error bars indicate S.D

peripheral vein of rats and tested the induction of neuroinflammation by cytokine measurements in the CSF (Fig. 1/ sub-study III). In fact, a striking increase of inflammatory cytokines in the CSF after peripheral injection of LPSderived EVs indicates that they passed the BBB in amounts that can induce neuroinflammation (Fig. 6).

Final proof that peripherally injected EVs can reach the brain was obtained by peripheral intravenous injection of fluorescently labeled EVs and their detection in CSF. LPStreatment or $\mathrm{HpX}$ had no effect on the permeability of the BBB for EVs (Fig. 7).
Table 1 Effect of physostigmine

\begin{tabular}{lllll}
\hline & & \multicolumn{3}{l}{ Reduction of cytokine release } \\
\cline { 3 - 5 } & & TNF $\alpha(\%)$ & IL1 $\beta(\%)$ & IL6 (\%) \\
\hline \multirow{2}{*}{ Exosome donors } & LPS & 26.5 & 20.9 & 26.9 \\
& HpX & 22.6 & 18.7 & 28.0 \\
Sub-study I & LPS & 26.2 & 22.2 & 23.7 \\
\multirow{3}{*}{ Sub-study II } & HpX & 28.6 & - & 24.4 \\
\multirow{3}{*}{ Sub-study III } & LPS & 29.6 & 18.8 & 22.9 \\
& HpX & 25.7 & 24.0 & 26.7 \\
& LPS & 21.2 & 22.3 & 29.7 \\
& HpX & 21.7 & - & 26.4 \\
\hline
\end{tabular}

Since the anticholinergic drug physostigmine was suggested to attenuate neuroinflammation induced by peripheral inflammation, we also tested whether the administration of the drug to the animals from which the vesicles were concentrated can alleviate the action of the LPS- and HpXderived EVs. An attenuating action of physostigmine was observed in all three sub-studies (Table 1).

\section{Discussion}

The LPS model of systemic inflammation that mimics many of the initial clinical features of sepsis is considered ideally suited to investigate the pathogenic effects of acute inflammation (Seemann et al. 2017). Likewise, the model of $\mathrm{HpX}$ is well appropriate to imitate a typical surgical intervention without an organ-specific deterioration (Plaschke et al. 2018b). Therefore, these models were used to collect EV pools that represent the two inflammatory states which were confirmed by blood analyses and the detection of strikingly increased proinflammatory cytokines in blood 
samples from the animals. As endotoxemia produced a considerably stronger inflammatory response as surgical stress, the two models not only represent two different causes of peripheral inflammation, but also mimic different levels of inflammation. Microglia regulates cytokines and inflammatory processes in the brain and the release of proinflammatory cytokines from these cells indicates the initiation of a neuroinflammatory reaction in the brain. In particular, the release of the proinflammatory cytokines TNF- $\alpha$, IL1 $\beta$ and IL6 is considered a major trigger of the inflammatory process (Becher et al. 2017). The human microglial HMC3 cell line provides a well-suited tool to analyze the potential of EV populations to activate a proinflammatory program in glial cells, since this cell line displays very similar functional properties, in particular with regard to proinflammatory cytokine release, as primary microglia cells (Dello Russo et al. 2018). Our results clearly proof that EVs concentrated from LPS- or HpX-animals are potent proinflammatory activators of microglia cells albeit with different efficiencies. The same amount of LPS-derived EVs induced a significantly stronger response than $\mathrm{HpX}$-derived EVs reflecting the different severity of inflammation in the donor animals. This may be explained either by the secretion of more potent proinflammatory EVs or just by a higher proportion of proinflammatory EVs in the total pool of EVs from the LPS-treated donors. Although our data suggested an EV-associated proinflammatory signaling that mediated the induction of neuroinflammation in both LPS-conditions (LPS, endotoxemia) and LPS-free conditions (HpX, peripheral surgical stress), we cannot rule out that residual LPS might contribute to the pronounced effects. However, it was reported that the half-life of LPS in the circulation of rodents is only about 2 to 4 min (Yao et al. 2016), making it very unlikely that residual LPS caused any observed effects. Further, using labeled LPS with radioactive-iodine (I-LPS), it was suggested that brain uptake of circulating I-LPS is so low that most effects of peripherally administered LPS are likely mediated through LPS receptors located outside of the BBB or triggered by other extracellular mediators (e.g., vesicles) (Banks and Robinson 2010).

Our results obtained by icv injection confirmed that LPS- or HpX-derived EVs are potent inducers of neuroinflammation in vivo. The findings of the cell culture experiments suggest that glial activation is also a major trigger in vivo. This is in line with a study where glial activation was assessed by immunohistochemistry in rats that were treated with EVs from endotoxemic donors ( $\mathrm{Li}$ et al. 2018). However, a contribution of other cells, e.g., astrocytes, cannot be excluded. Moreover, it cannot be ruled out that nonvesicular protein aggregates, soluble proteins or lipoprotein particles accounted for the proinflammatory responses to some extent. Evidence demonstrated that established EV isolation techniques (e.g., ultracentrifugation, ultrafiltration, size exclusion chromatography, precipitation, immunoaffinity) are not able to completely separate EVs from non-EV material, including lipoproteins (Brennan et al. 2020; Karimi et al. 2018; Simonsen 2017; Sódar et al. 2016; Takov et al. 2019; Webber and Clayton 2013; Yuana et al. 2014). Thus, lipoprotein contamination became an increasingly recognized obstacle/problem/topic in the field of EV research (Simonsen 2017; Thery et al. 2018). Although the EV isolation method itself plays a crucial role, no consensus on an optimal purification method has been achieved yet. It has been suggested that functional outcomes are dependent on the purification method (Takov et al. 2019). Here, we used differential ultracentrifugation to concentrate EVs from rat serum samples. Although the EV pellet will invariably encompass non-EV material and contaminating factors that co-sediment alongside EVs, ultracentrifugation is considered to be the most commonly used EV enrichment method (Gardiner et al. 2016). When compared to size exclusion chromatography, ultracentrifugation can lead to higher purity of EVs isolated from rat blood samples (Takov et al. 2019). In our study, the high-density lipoprotein (HDL) marker protein ApoA1 was found to be present in all EV preparations in almost similar concentrations, thereby excluding any treatment-associated effects caused by the apolipoprotein itself. It has been reported that some EVs carry lipoproteins, making it difficult to discriminate between lipoprotein contamination and EV-associated lipoproteins (Karimi et al. 2018; Sódar et al. 2016; van Niel et al. 2015). Future studies are required to investigate any possible biological impact of lipoproteins and other potential mediators that might trigger the EV-mediated proinflammatory signaling. This could be addressed in further experiments by combining multiple isolation methods, leading to a complete/improved/more stringent separation of extracellular particles and vesicles and purer EV populations (Gardiner et al. 2016; Karimi et al. 2018; Simonsen 2017; Takov et al. 2019). However, significant losses of EVs and high variability between EV preparations can be expected due to long procedures involving multiple handling steps. Novel EV isolation tools are in development that might help to overcome these limitations in the future.

EVs have already been described as potent vehicles that easily pass the BBB and deliver signaling molecules to the CNS (Das et al. 2019). Therefore, also proinflammatory EVs that are released into the peripheral blood stream should be able to transfer proinflammatory signals to the brain. Our results clearly confirm that EVs-when injected into the peripheral blood stream-can cross the BBB and transfer proinflammatory signals to the CNS. In future studies the question, which cargo molecules of the EVs carry the information that is transferred to the CNS, should be addressed. Top candidates are EV-packed miRNAs or proteins, since they have already been described as key players in sepsis and 
inflammatory tissue injury (Park et al. 2019). In circulating EVs of septic shock patients significant changes in a panel of 65 miRNAs compared to those of healthy controls were found and the pathways potentially influenced by these miRNAs were largely correlated with inflammatory response, oxidative damage, and cell-cycle checkpoint (Real et al. 2018). EV-associated miR-21, miR-126, miR-146, miR155 are examples of already confirmed regulators of inflammation in sepsis (Alexander et al. 2015; Chu et al. 2016; Pan et al. 2019; Song et al. 2017). Examples of proteins that represent EV-effective molecules in sepsis or systemic inflammation are caspase-3, SIP/S1PRS, neutrophil elastase (Genschmer et al. 2019; Moon et al. 2015; Park et al. 2018).

It has been shown that pharmacologic cholinesterase inhibition improves survival in experimental sepsis (Hofer et al. 2008). Accordingly the acetylcholinesterase inhibitor physostigmine attenuates neuroinflammation induced by surgical stress or endotoxemia (Kalb et al. 2013; Plaschke et al. 2018b, 2016; von Haefen et al. 2018). In our present study, physostigmine attenuated the pro-neuroinflammatory action of LPS- and HpX-derived EVs. Two different working points of physostigmine action can be envisioned. Activation of the nicotinic acetylcholine receptor alpha7 subunit is required for inflammatory activation of macrophages and thus, reduction of acetylcholine in the periphery by physostigmine may be responsible for the effect. On the other hand, cholinergic modulation of microglial activation (Shytle et al. 2004) has been shown to attenuate neuroinflammation that was primarily induced by peripheral inflammation (Terrando et al. 2015). Since peripheral inflammation as well as neuroinflammation is under the control of the vagus nerve-based inflammatory reflex both may be effective. Specific inhibition of cholinergic induction of a peripheral inflammation response reduced neuroinflammation in a surgical model, suggesting that the peripheral working point of physostigmine is involved in its anti-neuroinflammatory action (Kalb et al. 2013). Our results indicate that an effect of physostigmine on the EV cargo or the release of proinflammatory EVs in the periphery might at least partially contribute to its impact on neuroinflammation. In consequence, this would mean that peripheral cholinergic signaling influences EVmediated signaling from the periphery to the CNS. This should be confirmed and detailed in further studies.

Altogether our present study clearly indicates that EVs released in endotoxemia or by surgical stress have the potential to induce a proinflammatory reaction in microglia cells resulting in a release of inflammatory cytokines to the CSF. Since EVs primed by peripheral inflammation can easily translocate to the brain by passing through the BBB, EV proinflammatory signaling from the periphery to the CNS appears effective in the induction of neuroinflammation in sepsis or surgical stress. Peripheral cholinergic signaling could be involved in the initiation of the EV-mediated proinflammatory signaling. Detailed analysis of the cargo of proinflammatory EVs in already ongoing studies will help to understand the underlying mechanisms.

Acknowledgements The expert technical assistance of Roland Galmbacher, Klaus Stefan and Sigrun Himmelsbach is gratefully appreciated. We would also like to express our thanks to Dr. Ingrid Hausser and Ulrike Ganserer for their help with transmission electron microscopy. We also acknowledge financial support by Dr. Franz Köhler Chemie GmbH, Bensheim, Germany.

Funding Open Access funding enabled and organized by Projekt DEAL.

Open Access This article is licensed under a Creative Commons Attribution 4.0 International License, which permits use, sharing, adaptation, distribution and reproduction in any medium or format, as long as you give appropriate credit to the original author(s) and the source, provide a link to the Creative Commons licence, and indicate if changes were made. The images or other third party material in this article are included in the article's Creative Commons licence, unless indicated otherwise in a credit line to the material. If material is not included in the article's Creative Commons licence and your intended use is not permitted by statutory regulation or exceeds the permitted use, you will need to obtain permission directly from the copyright holder. To view a copy of this licence, visit http://creativecommons.org/licenses/by/4.0/.

\section{References}

Alexander M et al (2015) Exosome-delivered microRNAs modulate the inflammatory response to endotoxin. Nat Commun 6:7321. https://doi.org/10.1038/ncomms8321

Banks WA (2006) The blood-brain barrier in psychoneuroimmunology. Neurologic Clin 24:413-419. https://doi.org/10.1016/j.ncl. 2006.03.009

Banks WA, Robinson SM (2010) Minimal penetration of lipopolysaccharide across the murine blood-brain barrier. Brain Behav Immun 24:102-109. https://doi.org/10.1016/j.bbi.2009.09.001

Becher B, Spath S, Goverman J (2017) Cytokine networks in neuroinflammation. Nat Rev Immunol 17:49-59. https://doi.org/10. 1038/nri.2016.123

Becker A, Thakur BK, Weiss JM, Kim HS, Peinado H, Lyden D (2016) Extracellular vesicles in cancer: cell-to-cell mediators of metastasis. Cancer Cell 30:836-848. https://doi.org/10.1016/j.ccell.2016. 10.009

Bluthe RM et al (1994) Lipopolysaccharide induces sickness behaviour in rats by a vagal mediated mechanism. C R Acad Sci III 317:499-503

Brennan K et al (2020) A comparison of methods for the isolation and separation of extracellular vesicles from protein and lipid particles in human serum. Sci Rep 10:1039. https://doi.org/10.1038/ s41598-020-57497-7

Chen CC et al (2016) Elucidation of exosome migration across the blood-brain barrier model in vitro. Cell Mol Bioeng 9:509-529. https://doi.org/10.1007/s12195-016-0458-3

Chiu IM et al (2013) Bacteria activate sensory neurons that modulate pain and inflammation. Nature 501:52-57. https://doi.org/10. 1038/nature12479

Chu M et al (2016) Role of MiR-126a-3p in endothelial injury in endotoxic mice. Crit Care Med 44:e639-e650. https://doi.org/10.1097/ ccm.0000000000001629 
Das CK, Jena BC, Banerjee I, Das S, Parekh A, Bhutia SK, Mandal M (2019) Exosome as a novel shuttle for delivery of therapeutics across biological barriers. Mol Pharm 16:24-40. https://doi.org/ 10.1021/acs.molpharmaceut.8b00901

Dello Russo C et al (2018) The human microglial HMC3 cell line: where do we stand? A systematic literature review. J Neuroinflammation 15:259. https://doi.org/10.1186/s12974-018-1288-0

Gardiner C, Di Vizio D, Sahoo S, Théry C, Witwer KW, Wauben M, Hill AF (2016) Techniques used for the isolation and characterization of extracellular vesicles: results of a worldwide survey. J Extracell Vesicles 5:32945. https://doi.org/10.3402/jev.v5.32945

Genschmer KR et al (2019) Activated PMN exosomes: pathogenic entities causing matrix destruction and disease in the lung. Cell 176:113-126. https://doi.org/10.1016/j.cell.2018.12.002

Goehler LE, Gaykema RP, Hansen MK, Anderson K, Maier SF, Watkins LR (2000) Vagal immune-to-brain communication: a visceral chemosensory pathway. Auton Neurosci 85:49-59. https://doi.org/ 10.1016/s1566-0702(00)00219-8

Gupta A, Pulliam L (2014) Exosomes as mediators of neuroinflammation. J Neuroinflammation 11:68. https://doi.org/10.1186/ 1742-2094-11-68

Hofer S et al (2008) Pharmacologic cholinesterase inhibition improves survival in experimental sepsis. Crit Care Med 36:404-408. https://doi.org/10.1097/01.ccm.0b013e3181 $6208 \mathrm{~b} 3$

Kalb A et al (2013) Acetylcholinesterase inhibitors reduce neuroinflammation and -degeneration in the cortex and hippocampus of a surgery stress rat model. PLoS ONE 8:e62679. https://doi.org/ 10.1371/journal.pone.0062679

Karimi N et al (2018) Detailed analysis of the plasma extracellular vesicle proteome after separation from lipoproteins. Cell Mol Life Sci 75:2873-2886. https://doi.org/10.1007/s00018-018-2773-4

Kawahara H, Hanayama R (2018) The role of exosomes/extracellular vesicles in neural signal transduction. Biol Pharm Bull 41:1119_ 1125. https://doi.org/10.1248/bpb.b18-00167

$\mathrm{Li} \mathrm{JJ}$ et al (2018) In vivo evidence for the contribution of peripheral circulating inflammatory exosomes to neuroinflammation. J Neuroinflammation 15:8. https://doi.org/10.1186/s12974-017-1038-8

Liu W, Bai X, Zhang A, Huang J, Xu S, Zhang J (2019) Role of exosomes in central nervous system diseases. Front Mol Neurosci 12:240. https://doi.org/10.3389/fnmol.2019.00240

Mathieu M, Martin-Jaular L, Lavieu G, Théry C (2019) Specificities of secretion and uptake of exosomes and other extracellular vesicles for cell-to-cell communication. Nat Cell Biol 21:9-17. https://doi. org/10.1038/s41556-018-0250-9

Matsumoto J, Stewart T, Banks WA, Zhang J (2017) The transport mechanism of extracellular vesicles at the blood-brain barrier. Curr Pharm Des 23:6206-6214. https://doi.org/10.2174/13816 12823666170913164738

Moon HG, Cao Y, Yang J, Lee JH, Choi HS, Jin Y (2015) Lung epithelial cell-derived extracellular vesicles activate macrophage-mediated inflammatory responses via ROCK1 pathway. Cell Death Dis 6:e2016. https://doi.org/10.1038/cddis.2015.282

Pan T, Jia P, Chen N, Fang Y, Liang Y, Guo M, Ding X (2019) Delayed remote ischemic preconditioning confers renoprotection against septic acute kidney injury via exosomal miR-21. Theranostics 9:405-423. https://doi.org/10.7150/thno.29832

Park EJ, Appiah MG, Myint PK, Gaowa A, Kawamoto E, Shimaoka M (2019) Exosomes in sepsis and inflammatory tissue injury. Curr Pharm Des. https://doi.org/10.2174/13816128256661911161 25525

Park SJ et al (2018) Molecular mechanisms of biogenesis of apoptotic exosome-like vesicles and their roles as damage-associated molecular patterns. Proc Natl Acad Sci USA 115:E11721-E11730. https://doi.org/10.1073/pnas.1811432115
Pascual M, Ibanez F, Guerri C (2020) Exosomes as mediators of neuron-glia communication in neuroinflammation. Neural Regen Res 15:796-801. https://doi.org/10.4103/1673-5374.268893

Plaschke K, Do TQM, Uhle F, Brenner T, Weigand MA, Kopitz J (2018a) Ablation of the right cardiac vagus nerve reduces acetylcholine content without changing the inflammatory response during endotoxemia. Int J Mol Sci. https://doi.org/10.3390/ijms1 9020442

Plaschke K, Hoyer S (1993) Action of the diabetogenic drug streptozotocin on glycolytic and glycogenolytic metabolism in adult rat brain cortex and hippocampus. Int J Dev Neurosci 11:477-483. https://doi.org/10.1016/0736-5748(93)90021-5

Plaschke K, Schulz S, Rullof R, Weigand MA, Kopitz J (2018b) In-depth characterization of the neuroinflammatory reaction induced by peripheral surgery in an animal model. J Neural Transm (Vienna) 125:1487-1494. https://doi.org/10.1007/ s00702-018-1909-x

Plaschke K, Weigand MA, Fricke F, Kopitz J (2016) Neuroinflammation: effect of surgical stress compared to anaesthesia and effect of physostigmine. Neurol Res 38:397-405. https://doi.org/10.1080/ 01616412.2016.1173889

Poon DC, Ho YS, Chiu K, Wong HL, Chang RC (2015) Sickness: From the focus on cytokines, prostaglandins, and complement factors to the perspectives of neurons. Neurosci Biobehav Rev 57:30-45. https://doi.org/10.1016/j.neubiorev.2015.07.015

Rama Rao KV, Kielian T (2015) Neuron-astrocyte interactions in neurodegenerative diseases: role of neuroinflammation. Clin Exp Neuroimmunol 6:245-263. https://doi.org/10.1111/cen3.12237

Raposo G, Stahl PD (2019) Extracellular vesicles: a new communication paradigm? Nat Rev Mol Cell Biol. https://doi.org/10.1038/ s41580-019-0158-7

Real JM et al (2018) Exosomes from patients with septic shock convey miRNAs related to inflammation and cell cycle regulation: new signaling pathways in sepsis? Crit Care 22:68. https://doi.org/10. 1186/s13054-018-2003-3

Rochfort KD, Cummins PM (2015) The blood-brain barrier endothelium: a target for pro-inflammatory cytokines. Biochem Soc Trans 43:702-706. https://doi.org/10.1042/bst20140319

Seemann S, Zohles F, Lupp A (2017) Comprehensive comparison of three different animal models for systemic inflammation. J Biomed Sci 24:60. https://doi.org/10.1186/s12929-017-0370-8

Shytle RD et al (2004) Cholinergic modulation of microglial activation by alpha 7 nicotinic receptors. J Neurochem 89:337-343. https:// doi.org/10.1046/j.1471-4159.2004.02347.x

Simonsen JB (2017) What are we looking at? extracellular vesicles, lipoproteins, or both? Circ Res 121:920-922. https://doi.org/10. 1161/circresaha.117.311767

Sódar BW et al (2016) Low-density lipoprotein mimics blood plasmaderived exosomes and microvesicles during isolation and detection. Sci Rep 6:24316. https://doi.org/10.1038/srep24316

Song Y et al (2017) Exosomal miR-146a contributes to the enhanced therapeutic efficacy of interleukin-1beta-primed mesenchymal stem cells against sepsis. Stem Cells 35:1208-1221. https://doi. org/10.1002/stem.2564

Stahl PD, Raposo G (2018) Exosomes and extracellular vesicles: the path forward. Essays Biochem 62:119-124. https://doi.org/10. 1042/ebc20170088

Takov K, Yellon DM, Davidson SM (2019) Comparison of small extracellular vesicles isolated from plasma by ultracentrifugation or size-exclusion chromatography: yield, purity and functional potential. J Extracell Vesicles 8:1560809. https://doi.org/10.1080/ 20013078.2018.1560809

Terrando N et al (2015) Stimulation of the alpha7 nicotinic acetylcholine receptor protects against neuroinflammation after tibia fracture and endotoxemia in mice. Mol Med 20:667-675. https:// doi.org/10.2119/molmed.2014.00143 
Thery C et al (2018) Minimal information for studies of extracellular vesicles 2018 (MISEV2018): a position statement of the International society for extracellular vesicles and update of the MISEV2014 guidelines. J Extracell Vesicles 7:1535750. https:// doi.org/10.1080/20013078.2018.1535750

Upadhya D, Shetty AK (2019) Extracellular vesicles as therapeutics for brain injury and disease. Curr Pharm Des 25:3500-3505. https:// doi.org/10.2174/1381612825666191014164950

Van Deun J et al (2017) EV-TRACK: transparent reporting and centralizing knowledge in extracellular vesicle research. Nat Methods 14:228-232. https://doi.org/10.1038/nmeth.4185

van Niel G et al (2015) Apolipoprotein E regulates amyloid formation within endosomes of pigment. Cells Cell Rep 13:43-51. https:// doi.org/10.1016/j.celrep.2015.08.057

van Niel G, D'Angelo G, Raposo G (2018) Shedding light on the cell biology of extracellular vesicles. Nat Rev Mol Cell Biol 19:213228. https://doi.org/10.1038/nrm.2017.125

von Haefen C et al (2018) Physostigmine restores impaired autophagy in the rat hippocampus after surgery stress and LPS treatment. $\mathrm{J}$ Neuroimmune Pharmacol 13:383-395. https://doi.org/10.1007/ s11481-018-9790-9

Webber J, Clayton A (2013) How pure are your vesicles? J Extracell Vesicles 10:2. https://doi.org/10.3402/jev.v2i0.19861
Witwer KW, Théry C (2019) Extracellular vesicles or exosomes? On primacy, precision, and popularity influencing a choice of nomenclature. J Extracell Vesicles 8:1648167. https://doi.org/10.1080/ 20013078.2019.1648167

Wortzel I, Dror S, Kenific CM, Lyden D (2019) Exosome-mediated metastasis: communication from a distance. Dev Cell 49:347-360. https://doi.org/10.1016/j.devcel.2019.04.011

Wuerfel E, Infante-Duarte C, Glumm R, Wuerfel JT (2010) Gadofluorine M-enhanced MRI shows involvement of circumventricular organs in neuroinflammation. J Neuroinflammation 7:70. https:// doi.org/10.1186/1742-2094-7-70

Yao Z et al (2016) Blood-borne lipopolysaccharide is rapidly eliminated by liver sinusoidal endothelial cells via high-density lipoprotein. J Immunol 197:2390-2399. https://doi.org/10.4049/ jimmunol.1600702

Yuana Y, Levels J, Grootemaat A, Sturk A, Nieuwland R (2014) Coisolation of extracellular vesicles and high-density lipoproteins using density gradient ultracentrifugation. J Extracell Vesicles 8:3. https://doi.org/10.3402/jev.v3.23262

Publisher's Note Springer Nature remains neutral with regard to jurisdictional claims in published maps and institutional affiliations. 